\title{
Improvement Dynamics in Games with Strategic Complementarities*
}

\author{
Nikolai S. Kukushkin ${ }^{\dagger} \quad$ Satoru Takahashi ${ }^{\ddagger} \quad$ Tetsuo Yamamori ${ }^{\S}$
}

\begin{abstract}
In a finite game with strategic complementarities, every strategy profile is connected to a Nash equilibrium with a finite individual improvement path. If, additionally, the strategies are scalar, then every strategy profile is connected to a Nash equilibrium with a finite individual best response improvement path.
\end{abstract}

Key words: Nash equilibrium, Better response dynamics, Best response dynamics, Game with strategic complementarities

*The first author acknowledges financial support from the Russian Foundation for Basic Research (grant 02-01-00854) and from a presidential grant for the state support of the leading scientific schools (NSh-1843.2003.01); he also thanks Universidad Carlos III de Madrid, Departamento de Economía, and personally Francisco Marhuenda for their hospitality. The other authors would like to thank Michihiro Kandori, Akihiko Matsui, Jim Friedman, Claudio Mezzetti, and Federico Echenique for helpful comments. All the three thank an anonymous referee and the Editor of this journal for bringing us together and suggesting the merger of two independent works.

${ }^{\dagger}$ Russian Academy of Sciences, Dorodnicyn Computing Center, 40, Vavilova, Moscow 119991 Russian Federation. E-mail: ququ@ccas.ru

${ }^{\ddagger}$ Department of Economics, Harvard University, Cambridge, MA 02138, USA. E-mail: stakahas@fas.harvard.edu

${ }^{\S}$ Graduate School of Economics, University of Tokyo, 7-3-1 Hongo, Bunkyo-ku, Tokyo 113-0033, JAPAN. E-mail: ee27004@mail.ecc.u-tokyo.ac.jp 


\section{Introduction}

Learning and adaptation in strategic games is an important and interesting topic, attracting much attention (Milgrom and Roberts, 1990, 1991; Young, 1993; Kandori and Rob, 1995; Monderer and Shapley, 1996; Milchtaich, 1996; Fudenberg and Levine, 1998; Friedman and Mezzetti, 2001; Kukushkin, 2004). Quite often, the analysis of sophisticated scenarios involving random moves crucially depends on simple properties of the game, expressed in terms of improvement paths. It is essential, therefore, to understand when we can expect which property to hold.

For instance, when Young (1993) found the importance of the condition that every strategy profile should be connected to a Nash equilibrium with a best response path, he was unable to point out a single natural class of games where the property had been established. Since then, much work has been done; in particular, that property holds in every finite potential game as defined by Monderer and Shapley (1996), who provide quite a list of such games. Still, we cannot say that the subject is thoroughly investigated.

Convergence properties of improvement dynamics in games with strategic complementarities have been considered since the very beginning: Topkis (1979) and Vives (1990) showed that every best response improvement path starting from a certain region in the outcomes space eventually reaches a Nash equilibrium. Milgrom and Roberts (1990) studied rather general adaptive scenarios without explicitly considering their convergence. Kandori and Rob (1995) established the convergence to a Nash equilibrium of every best response path in every finite, symmetric, and strictly supermodular game with scalar strategies; Kukushkin (2004) established the same property of best response improvement paths in games with additive aggregation. Friedman and Mezzetti (2001) showed that every strategy profile in a finite game with strategic complementarities and onedimensional strategy sets can be connected to a Nash equilibrium with an improvement path.

This paper strengthens the last result considerably: it turns out that Friedman and Mezzetti's statement holds in a multidimensional context as well, whereas their conditions ensure the possibility to reach a Nash equilibrium with a best response improvement path. Both extensions are more than purely technical hair-splitting. Although most games with strategic complementarities in the literature have scalar strategies, multidimensional models are also important: e.g., in the context of industrial organization, it is natural for a firm to choose several parameters (Vives, 2003, Section 4.4). The difference between better response and best response dynamics is crucial, e.g., for the scenario of Young (1993) mentioned above. 
Strategic complementarities are interpreted in the broadest sense available in the literature: we assume single crossing (Milgrom and Shannon, 1994) and pseudosupermodularity (Agliardi, 2000) conditions. The proofs use the technique of binary relations suggested in Kukushkin $(1999,2004)$.

The attention here is restricted to finite games. Similar results can be obtained for the infinite (topological) case too; however, the proofs need rather heavy tools such as the notion of improvement paths parameterized with transfinite numbers, outlined in Kukushkin (2000).

Section 2 contains basic formal definitions. Section 3 reproduces conditions usually associated with the term "strategic complementarities." Section 4 contains the main theorems and examples showing the impossibility of easy generalizations. The proofs are deferred to Section 5 .

\section{Basic Notions}

A strategic game is defined by a finite set of players $N$, and strategy sets $X_{i}$ and ordinal utility functions $u_{i}$ on $X=\prod_{i \in N} X_{i}$ for all $i \in N$. In this paper, we assume that each $X_{i}$ is a finite set.

For every player $i \in N$, the best response correspondence $R_{i}(\cdot)$ is defined in the usual way:

$$
R_{i}\left(x_{-i}\right)=\left\{y_{i} \in X_{i} \mid \forall z_{i} \in X_{i}\left[u_{i}\left(y_{i}, x_{-i}\right) \geq u_{i}\left(z_{i}, x_{-i}\right)\right]\right\},
$$

where $x_{-i} \in X_{-i}=\prod_{j \in N \backslash\{i\}} X_{j}$.

We introduce a number of binary relations on $X(y, x \in X$ and $i \in N)$ :

$$
\begin{gathered}
y \triangleright_{i} x \Longleftrightarrow\left[y_{-i}=x_{-i} \& u_{i}(y)>u_{i}(x)\right] ; \\
y \triangleright x \Longleftrightarrow \exists i \in N\left[y \triangleright_{i} x\right] ; \\
y \triangleright_{i}^{*} x \Longleftrightarrow\left[y_{-i}=x_{-i} \& u_{i}(y)>u_{i}(x) \& y_{i} \in R_{i}\left(x_{-i}\right)\right] ; \\
y \triangleright^{*} x \Longleftrightarrow \exists i \in N\left[y \triangleright_{i}^{*} x\right] .
\end{gathered}
$$

A strategy profile $x \in X$ is a Nash equilibrium if and only if $x$ is a maximizer for $\triangleright$, i.e., if $y \triangleright x$ is impossible for any $y \in X$; equivalently, $x \in X$ is a Nash equilibrium if and only if $x$ is a maximizer for $\triangleright^{*}$.

An (individual) improvement path is a finite or infinite sequence $\left\{x^{k}\right\}_{k=0,1, \ldots, \text { such that }}$ $x^{k+1} \triangleright x^{k}$ whenever $k \geq 0$ and $x^{k+1}$ is defined. A best response improvement path is a 
finite or infinite sequence $\left\{x^{k}\right\}_{k=0,1, \ldots}$, such that $x^{k+1} \triangleright^{*} x^{k}$ whenever $k \geq 0$ and $x^{k+1}$ is defined.

Putting together the terminology of Monderer and Shapley (1996), Milchtaich (1996), and Friedman and Mezzetti (2001), we introduce the following definitions.

A game has the finite improvement path (FIP) property if there exists no infinite improvement path. A game has the finite best response improvement path (FBRP) property if there exists no infinite best response improvement path. A game has the weak FIP property if, for every $x \in X$, there exists a finite improvement path $\left\{x^{0}, \ldots, x^{m}\right\}$ such that $x^{0}=x$ and $x^{m}$ is a Nash equilibrium, i.e., if every strategy profile is connected to a Nash equilibrium with a finite improvement path. A game has the weak FBRP property if, for every $x \in X$, there exists a finite best response improvement path $\left\{x^{0}, \ldots, x^{m}\right\}$ such that $x^{0}=x$ and $x^{m}$ is a Nash equilibrium.

It is easy to see that the following chain of implications holds:

$$
\mathrm{FIP} \Rightarrow \mathrm{FBRP} \Rightarrow \text { weak FBRP } \Rightarrow \text { weak FIP. }
$$

Each of these properties characterizes the global convergence property in a corresponding class of learning and adaptive dynamics. A stationary Markov process on $X$ is said to be a better-reply (respectively best-reply) dynamic if (1) for each strategy profile $x$ that is not a Nash equilibrium, there exists $x^{\prime}$ such that $x^{\prime} \triangleright x$ (respectively $x^{\prime} \triangleright^{*} x$ ) and the one step transition probability from $x$ to $x^{\prime}$ is positive, and (2) every Nash equilibrium is absorbing. It is easy to show that a game has the FIP (respectively FBRP) property if and only if, under any better-reply (respectively best-reply) dynamic, the sequence of strategy profiles from any initial strategy profile almost surely converges to a Nash equilibrium. Alternatively, if we assume those dynamics to have full support, i.e., for any $x^{\prime}$ such that $x^{\prime} \triangleright x$ (respectively $x^{\prime} \triangleright^{*} x$ ), the one step transition probability from $x$ to $x^{\prime}$ is positive, then we obtain the weak versions of the above statements. See Friedman and Mezzetti (2001), Kandori and Rob (1995), and Milchtaich (1996) for examples of betterand best-reply dynamics.

\section{Games with Strategic Complementarities}

For the term to be applicable to a game, the latter must satisfy several requirements, which can be formalized in somewhat different ways.

First, we assume that each $X_{i}$ is a lattice; therefore, $X$ and every $X_{-i}$ are lattices too. 
Second, there must be a complementarity condition on strategies of different players. The basic choice is between Topkis's (1979) cardinal increasing differences condition:

$$
\left[y_{i} \geq x_{i} \& y_{-i} \geq x_{-i}\right] \Rightarrow\left[u_{i}(y)-u_{i}\left(x_{i}, y_{-i}\right) \geq u_{i}\left(y_{i}, x_{-i}\right)-u_{i}(x)\right]
$$

and Milgrom and Shannon's (1994) ordinal single crossing condition:

$$
\left[y_{i} \geq x_{i} \& y_{-i} \geq x_{-i}\right] \Rightarrow\left[\operatorname{sign}\left(u_{i}(y)-u_{i}\left(x_{i}, y_{-i}\right)\right) \geq \operatorname{sign}\left(u_{i}\left(y_{i}, x_{-i}\right)-u_{i}(x)\right)\right],
$$

where $i \in N, x_{i}, y_{i} \in X_{i}, x_{-i}, y_{-i} \in X_{-i}$, and $\operatorname{sign}(t)$ is -1 if $t<0,0$ if $t=0$, and 1 if $t>0$ (although subtraction is used in the second definition, the property itself is purely ordinal).

Finally, if strategies of a given player $i$ are multidimensional, there must be a complementarity condition on different dimensions. Here we have even three versions: the cardinal supermodularity condition

$$
u_{i}\left(x_{i} \vee y_{i}, z_{-i}\right)-u_{i}\left(x_{i}, z_{-i}\right) \geq u_{i}\left(y_{i}, z_{-i}\right)-u_{i}\left(x_{i} \wedge y_{i}, z_{-i}\right)
$$

the ordinal quasisupermodularity condition (Milgrom and Shannon, 1994)

$$
\operatorname{sign}\left(u_{i}\left(x_{i} \vee y_{i}, z_{-i}\right)-u_{i}\left(x_{i}, z_{-i}\right)\right) \geq \operatorname{sign}\left(u_{i}\left(y_{i}, z_{-i}\right)-u_{i}\left(x_{i} \wedge y_{i}, z_{-i}\right)\right)
$$

the, also ordinal, pseudosupermodularity condition (Agliardi, 2000)

$$
\begin{aligned}
& \operatorname{sign}\left(\max \left\{u_{i}\left(x_{i} \vee y_{i}, z_{-i}\right)-u_{i}\left(x_{i}, z_{-i}\right), u_{i}\left(x_{i} \vee y_{i}, z_{-i}\right)-u_{i}\left(y_{i}, z_{-i}\right)\right\}\right) \geq \\
& \operatorname{sign}\left(\max \left\{u_{i}\left(x_{i}, z_{-i}\right)-u_{i}\left(x_{i} \wedge y_{i}, z_{-i}\right), u_{i}\left(y_{i}, z_{-i}\right)-u_{i}\left(x_{i} \wedge y_{i}, z_{-i}\right)\right\}\right) .
\end{aligned}
$$

Again, $i \in N, x_{i}, y_{i} \in X_{i}$, and $z_{-i} \in X_{-i} ; \operatorname{sign}(t)$ has the same meaning as above.

The following implications are easy to check: $(3.1) \Rightarrow(3.2) ;(3.3) \Rightarrow(3.4) \Rightarrow(3.5)$.

Lemma 3.1. If a utility function $u_{i}$ satisfies (3.2) and (3.5) for each $x_{i}, y_{i} \in X_{i}$ and $x_{-i}, y_{-i}, z_{-i} \in X_{-i}$, then

$$
\left[y_{-i} \geq x_{-i} \& y_{i} \in R_{i}\left(y_{-i}\right) \& x_{i} \in R_{i}\left(x_{-i}\right)\right] \Rightarrow\left[y_{i} \vee x_{i} \in R_{i}\left(y_{-i}\right) \& y_{i} \wedge x_{i} \in R_{i}\left(x_{-i}\right)\right] .
$$

The statement means that $R_{i}\left(x_{-i}\right)$ is a sublattice of $X_{i}$ (pick $y_{-i}=x_{-i}$ ) and $R_{i}(\cdot)$ is increasing w.r.t. the strong set order defined by Veinott (see Topkis, 1979).

Proof. Indeed, $x_{i} \in R_{i}\left(x_{-i}\right)$ implies $u_{i}(x) \geq u_{i}\left(x_{i} \wedge y_{i}, x_{-i}\right)$ and $u_{i}(x) \geq u_{i}\left(y_{i}, x_{-i}\right)$, hence, by (3.5), $u_{i}\left(x_{i} \vee y_{i}, x_{-i}\right) \geq u_{i}\left(y_{i}, x_{-i}\right)$, hence, by (3.2), $u_{i}\left(x_{i} \vee y_{i}, y_{-i}\right) \geq u_{i}(y)$, hence $x_{i} \vee y_{i} \in R_{i}\left(y_{-i}\right)$. On the other hand, $y_{i} \in R_{i}\left(y_{-i}\right)$ implies that $u_{i}(y) \geq u_{i}\left(x_{i} \vee y_{i}, y_{-i}\right)$, hence, by (3.2), $u_{i}\left(y_{i}, x_{-i}\right) \geq u_{i}\left(x_{i} \vee y_{i}, x_{-i}\right)$, hence, by (3.5), $u_{i}\left(x_{i} \wedge y_{i}, x_{-i}\right) \geq u_{i}(x)$, hence $x_{i} \wedge y_{i} \in R_{i}\left(x_{-i}\right)$. 
Remark. The lemma is obviously inspired by Proposition 3 of Agliardi (2000), but is formally independent of it.

All the above properties admit strict versions; we only use one of them. A utility function $u_{i}$ is strictly pseudosupermodular if it satisfies (3.5) and, besides, whenever $x_{i}$ and $y_{i}$ are incomparable and the right hand side of (3.5) is 0 , the left hand side is 1 (when $x_{i}$ and $y_{i}$ are comparable, (3.5) becomes an equality).

Remark. In a sense, a generic pseudosupermodular function on a finite set is strictly pseudosupermodular.

Lemma 3.2. If a utility function $u_{i}$ is strictly pseudosupermodular and satisfies (3.2) for each $x_{i}, y_{i} \in X_{i}$ and $x_{-i}, y_{-i} \in X_{-i}$, then

$$
\left[y_{-i} \geq x_{-i} \& y_{i} \in R_{i}\left(y_{-i}\right) \& x_{i} \in R_{i}\left(x_{-i}\right)\right] \Rightarrow\left[y_{i} \leq x_{i} \text { or } y_{i} \geq x_{i}\right]
$$

(i.e., $R_{i}\left(x_{-i}\right) \cup R_{i}\left(y_{-i}\right)$ is a chain).

Proof. Suppose to the contrary that $x_{i}$ and $y_{i}$ are incomparable. Lemma 3.1 implies $x_{i} \wedge y_{i} \in R_{i}\left(x_{-i}\right)$; therefore, the right hand side of (3.5) with $z_{-i}=x_{-i}$ is 0 , hence the left hand side must be 1 , i.e., $u_{i}\left(x_{i} \vee y_{i}, x_{-i}\right)>u_{i}\left(y_{i}, x_{-i}\right)$. Now (3.2) implies $u_{i}\left(x_{i} \vee y_{i}, y_{-i}\right)>$ $u_{i}(y)$, contradicting $y_{i} \in R_{i}\left(y_{-i}\right)$.

\section{Theorems and Examples}

Theorem 1. If a game satisfies (3.2) and (3.5), then it has the weak FIP property.

If all strategies are scalar (i.e., each $X_{i}$ is a chain), then (3.5) is satisfied automatically and may be dropped. Otherwise, it is essential as the following example shows.

Example 1. Let us consider a two person game with $X_{1}=X_{2}=\{(0,0),(0,1),(1,0)$, $(1,1)\} \subseteq \mathbb{R}^{2}$ (with the standard order) and utilities described by these "matrices" (where player 1 chooses a $2 \times 2$ "block," and player 2 a position in the block; the axes are directed upwards and to the right):

$$
\begin{aligned}
& {\left[\begin{array}{ll}
\frac{(9,8)}{(6,6)} & (6,6) \\
(8,9)
\end{array}\right] \quad\left[\begin{array}{ll}
(6,6) & (7,7) \\
(2,2) & (6,6)
\end{array}\right]} \\
& {\left[\begin{array}{ll}
(6,6) & (2,2) \\
(7,7) & (6,6)
\end{array}\right] \quad\left[\begin{array}{ll}
(8,9) & (6,6) \\
(6,6) & \underline{(9,8)}
\end{array}\right]}
\end{aligned}
$$


Although the statement of Lemma 3.1 (as well as the condition (3.1)) holds for the game, the condition (3.5) does not. There exist two Nash equilibria: $\langle(0,0),(0,0)\rangle$ and $\langle(1,1),(1,1)\rangle$. However, no strategy profile from $\{(0,1),(1,0)\} \times\{(0,1),(1,0)\}$ can be connected to an equilibrium with an improvement path.

Theorem 2. If each strategy set $X_{i}$ is a chain, except one, say $X_{1}$, which is a lattice, (3.2) holds for all $i \in N$, and (3.5) for $i=1$, then the game has the weak FBRP property.

Example 2. Let us consider a two person game with the same strategy sets as in Example 1, but with different utilities (depicted under the same conventions):

$$
\begin{aligned}
& {\left[\begin{array}{ll}
(\underline{4}, 0) & (3,3) \\
(2,2) & (0, \underline{4})
\end{array}\right] \quad\left[\begin{array}{ll}
(3,2) & (\underline{6}, \underline{\underline{6}}) \\
(0,0) & (2,3)
\end{array}\right]} \\
& {\left[\begin{array}{ll}
(2,3) & (0,0) \\
(\underline{6}, \underline{6}) & (3,2)
\end{array}\right] \quad\left[\begin{array}{ll}
(0, \underline{4}) & (2,2) \\
(3,3) & (\underline{4}, 0)
\end{array}\right]}
\end{aligned}
$$

The game satisfies both conditions of Theorem 1; actually, even strict versions of (3.1) and (3.3). However, no strategy profile from $\{(0,1),(1,0)\} \times\{(0,1),(1,0)\}$ can be connected to an equilibrium with a best response improvement path. (The best responses of both players are underlined in the "matrix.")

Theorem 3. If, in a two person game, $X_{1}$ is a lattice, $X_{2}$ is a chain, (3.2) holds for both $i$, and $u_{1}$ is strictly pseudosupermodular, then the game has the FBRP property.

Remark. If both $X_{i}$ are chains, the FBRP property immediately follows from Theorem 1 of Kukushkin (2004): condition (2) there is even weaker than our (3.2).

Example 3. Let us consider a two person game with $X_{1}=\{(0,0),(0,1),(1,0),(1,1)\} \subseteq$ $\mathbb{R}^{2}, X_{2}=\{0,1\} \subseteq \mathbb{R}$ (with the standard order) and utilities described by these "matrices" (where player 1 chooses a block enclosed by brackets and player 2 a position in the block; the axes are directed upwards and to the right):

$$
\begin{aligned}
& {\left[\begin{array}{ll}
(2, \underline{\underline{3}}) & (\underline{3}, 2)]
\end{array}\left[\begin{array}{ll}
(2,2) & (\underline{3}, \underline{\underline{3}})]
\end{array}\right]\right.} \\
& {\left[\begin{array}{ll}
(\underline{3}, \underline{\underline{3}}) & (2,2)]
\end{array}\right]\left[\begin{array}{ll}
(\underline{3}, 2) & (2, \underline{\underline{3}})
\end{array}\right]}
\end{aligned}
$$

The condition (3.1) holds for both players; $u_{1}$ is supermodular, but not strictly pseudosupermodular. There is a best response improvement cycle:

$$
\langle(1,0), 1\rangle \stackrel{1}{\rightarrow}\langle(0,1), 1\rangle \stackrel{2}{\rightarrow}\langle(0,1), 0\rangle \stackrel{1}{\rightarrow}\langle(1,0), 0\rangle \stackrel{2}{\rightarrow}\langle(1,0), 1\rangle .
$$


Example 4. Let us consider a three person game with $X_{i}=\{0,1\}$ for all $i$ and utilities $u_{i}(x)=-\left|x_{i}-x_{\varphi(i)}\right|$, where $\varphi(1)=2, \varphi(2)=3, \varphi(3)=1$. The condition (3.1) obviously holds; all strategy sets are chains. However, there is a best response improvement cycle:

$$
\langle 1,0,1\rangle \stackrel{1}{\rightarrow}\langle 0,0,1\rangle \stackrel{2}{\rightarrow}\langle 0,1,1\rangle \stackrel{3}{\rightarrow}\langle 0,1,0\rangle \stackrel{1}{\rightarrow}\langle 1,1,0\rangle \stackrel{2}{\rightarrow}\langle 1,0,0\rangle \stackrel{3}{\rightarrow}\langle 1,0,1\rangle .
$$

Finally, the example from Section 7.6 of Kukushkin (2004) shows that we cannot expect the FIP property even in a two person game with scalar strategies and strictly increasing differences.

\section{Proofs}

\subsection{Proof of Theorem 1}

To produce an improvement path from an arbitrary strategy profile to an equilibrium, we impose the following rules: (1) If, at a current profile, there exist profitable deviations upwards (i.e., with $y_{i}>x_{i}$ ), one of them must be chosen. (2) Otherwise, one of the most profitable (for the deviating player) deviations downwards (with $y_{i}<x_{i}$ ) must be chosen.

(3) If neither (1) nor (2) can be applied, stop. It turns out that a path abiding by these rules cannot cycle and only stops at a Nash equilibrium.

Let us introduce some notation:

$$
\begin{gathered}
X^{+}=\{x \in X \mid \quad \exists y \in X[y>x \& y \triangleright x]\} ; X^{-}=X \backslash X^{+} ; \\
R_{i}^{-}(x)=\left\{y_{i} \in X_{i} \mid \quad y_{i} \leq x_{i} \& \forall z_{i} \in X_{i}\left[z_{i} \leq x_{i} \Rightarrow u_{i}\left(y_{i}, x_{-i}\right) \geq u_{i}\left(z_{i}, x_{-i}\right)\right]\right\} ; \\
y \triangleright_{i} x \Longleftrightarrow\left[y \triangleright_{i} x \&\left(y_{i}>x_{i} \text { or }\left[x \in X^{-} \& y_{i} \in R_{i}^{-}(x)\right]\right)\right] ; \\
y \bowtie x \Longleftrightarrow \exists i \in N\left[y \triangleright_{i} x\right] ; \\
y \succ x \Longleftrightarrow\left[\left(y \in X^{-} \& x \in X^{+}\right) \text {or }\left(x, y \in X^{+} \& y>x\right) \text { or }\left(x, y \in X^{-} \& y<x\right)\right] .
\end{gathered}
$$

It is easy to check that $\succ$ is irreflexive and transitive. By definition, $y \bowtie x \Rightarrow y \triangleright x$; an improvement path abides by the rules if and only if $x^{k+1} \bowtie x^{k}$ for all relevant $k$ and the path either is infinite or ends at a maximizer for $\triangleright$.

Lemma 5.1.1. If $y \bowtie x$, then $y \succ x$.

Proof. The only point worth discussing is the incompatibility of $x \in X^{-}$and $y \bowtie_{j} x$ with $y \in X^{+}$. Suppose the contrary: there are $i \in N$ and $z_{i}>y_{i}$ such that

$$
u_{i}\left(z_{i}, y_{-i}\right)>u_{i}(y)
$$


Since $x \in X^{-}, y_{j}<x_{j}$. If $i \neq j$, then $y_{i}=x_{i}$ and $y_{-i}<x_{-i}$. Now (5.1) and (3.2) imply $u_{i}\left(z_{i}, x_{-i}\right)>u_{i}(x)$, contradicting $x \in X^{-}$.

Thus, we are led to $i=j$, hence $y_{-i}=x_{-i}$. Now $y_{i} \in R_{i}^{-}(x)$ implies $u_{i}(y) \geq$ $u_{i}\left(z_{i} \wedge x_{i}, x_{-i}\right)$, hence, by (5.1) and (3.5), $u_{i}\left(z_{i} \vee x_{i}, x_{-i}\right)>u_{i}(x)$, hence $z_{i} \vee x_{i}>x_{i}$, again contradicting $x \in X^{-}$.

Lemma 5.1.2. If $x \in X$ is a maximizer for $\bowtie$, then $x$ is a Nash equilibrium (i.e., a maximizer for $\triangleright$ ).

Proof. Suppose the contrary: $x \in X$ is a maximizer for $\bowtie$ (i.e., there is no profitable deviation either upwards or downwards), but not a Nash equilibrium, i.e., there exist $i \in N$ and $y_{i} \in X_{i}$ such that $u_{i}\left(y_{i}, x_{-i}\right)>u_{i}(x)$ (then $y_{i}$ must be incomparable with $x_{i}$ ). We have $x_{i} \wedge y_{i}<x_{i}$, hence $u_{i}\left(x_{i} \wedge y_{i}, x_{-i}\right) \leq u_{i}(x)<u_{i}\left(y_{i}, x_{-i}\right)$; similarly, $x_{i} \vee y_{i}>x_{i}$, hence $u_{i}\left(x_{i} \vee y_{i}, x_{-i}\right) \leq u_{i}(x)<u_{i}\left(y_{i}, x_{-i}\right)$. Now we have a contradiction with (3.5).

Lemma 5.1.1 implies that every improvement path abiding by the rules ends at a maximizer for $\triangleright$. Lemma 5.1.2 implies that the maximizer is a Nash equilibrium. Theorem 1 is proven.

\subsection{Proof of Theorem 2}

We will reason similarly to the proof of Theorem 1, considering best response improvement paths abiding by the following rules: (1) If, at a current profile, $x_{1} \notin R_{1}\left(x_{-1}\right)$, then a best response improvement by player 1 must be implemented, upwards (with $y_{1}>x_{1}$ ) if possible; otherwise, we choose any best response improvement by player 1 if there is a player $i \neq 1$ with a possible best response improvement upwards $\left(y_{i}>x_{i}\right)$, but try to find a best response improvement $y_{1}<x_{1}$ first if there is no such $i$. (2) If $x_{1} \in R_{1}\left(x_{-1}\right)$ and there exist best response improvements upwards (with $y_{i}>x_{i}$ ), one of them must be chosen. (3) Otherwise, one of the best response improvements downwards (with $y_{i}<x_{i}$ ) must be chosen. (4) If none of (1), (2), (3) can be applied, stop. Again, a path abiding by the rules cannot cycle and only stops at a Nash equilibrium.

Let us introduce some notation:

$$
\begin{gathered}
X^{1}=\left\{x \in X \mid \exists y>x\left[y \triangleright^{*}{ }_{1} x\right] \text { or }\left(x_{1} \in R_{1}\left(x_{-1}\right) \& \exists y>x\left[y \triangleright^{*} x\right]\right)\right\} ; \\
X^{2}=\left\{x \in X \mid \nexists y>x\left[y \triangleright^{*} x\right] \&\left(x_{1} \in R_{1}\left(x_{-1}\right) \text { or } \exists y<x\left[y \triangleright_{1}^{*} x\right]\right)\right\} ; \\
X^{0}=X \backslash\left(X^{1} \cup X^{2}\right) ;
\end{gathered}
$$




$$
\begin{aligned}
& y \triangleright_{i} x \Longleftrightarrow\left[y \triangleright _ { i } ^ { * } x \& \left(x_{1} \notin R_{1}\left(x_{-1}\right) \Rightarrow\right.\right. \\
& \quad(x \in 1) \& \\
& \left.\left.y \bowtie x \Longleftrightarrow X^{1} \Rightarrow y_{i}>x_{i}\right) \&\left(x \in X^{2} \Rightarrow y_{i}<x_{i}\right)\right] ; \\
& y i \in N\left[y \triangleright_{i} x\right] ; \\
& y \succ x \Longleftrightarrow\left[\left(y \in X^{k} \& x \in X^{h} \& k>h\right)\right. \text { or } \\
& \left.\left(x, y \in X^{1} \& y>x\right) \text { or }\left(x, y \in X^{2} \& y<x\right)\right] .
\end{aligned}
$$

It is easy to check that $\succ$ is irreflexive and transitive. By definition, $y \bowtie x \Rightarrow y \triangleright^{*} x$; a best response improvement path abides by the rules if and only if $x^{k+1} \bowtie x^{k}$ for all relevant $k$ and the path either is infinite or ends at a maximizer for $\bowtie$.

The next lemma describes, in a compact way, the following properties of our paths. From $x \in X^{0}$, we pass to $X^{1} \cup X^{2}$ and never return back. From $x \in X^{1}$, we always go upwards. From $x \in X^{2}$, we always go downwards and never come to $X^{0} \cup X^{1}$.

Lemma 5.2.1. If $y \triangleright_{j} x(j \in N)$, then $y \succ x$.

Proof. (A) Let $x \in X^{2}$, then $y<x$, so $y \succ x$ provided $y \in X^{2}$. Suppose, to the contrary, that there are $i \in N$ and $z_{i}>y_{i}$ such that

$$
y_{i} \notin R_{i}\left(y_{-i}\right) \ni z_{i}
$$

If $i=j$ (hence $y_{-i}=x_{-i}$ ), then $y_{i} \in R_{i}\left(y_{-i}\right)$ and (5.2) is impossible. Thus, $i \neq j$, hence $y_{i}=x_{i}<z_{i}$ and $y_{-i}<x_{-i}$. Supposing $y_{i} \in R_{i}\left(x_{-i}\right)$, we would have $y_{i}=y_{i} \wedge z_{i} \in R_{i}\left(y_{-i}\right)$ by Lemma 3.1, contradicting (5.2); therefore, $y_{i}=x_{i} \notin R_{i}\left(x_{-i}\right)$. Let $x_{i}^{\prime} \in R_{i}\left(x_{-i}\right)$; by Lemma 3.1 and (5.2), $y_{i}^{\prime}=x_{i}^{\prime} \vee z_{i} \in R_{i}\left(x_{-i}\right)$. Since $y_{i}^{\prime} \geq z_{i}>y_{i}$ and $\left(y_{i}^{\prime}, x_{-i}\right) \triangleright^{*} x$, we have $x \notin X^{2}$, contradicting our assumption. So the first condition in the definition of $X^{2}$ is fulfilled.

If $y_{1} \in R_{1}\left(y_{-1}\right)$, then $y \in X^{2}$; otherwise, $j \neq 1$, hence $y_{1}=x_{1} \in R_{1}\left(x_{-1}\right)$ and $y_{-1}<x_{-1}$. Picking $y_{1}^{\prime} \in R_{1}\left(y_{-1}\right)$, we denote $z_{1}=y_{1}^{\prime} \wedge y_{1}$; now $z_{1}<y_{1}$ and $z_{1} \in R_{1}\left(y_{-1}\right)$ by Lemma 3.1. Therefore, $\left(z_{1}, y_{-1}\right) \triangleright^{*}{ }_{1} y$, hence $y \in X^{2}$.

(B) It is easy to check that $x \in X^{0}$ implies $x_{1} \notin R_{1}\left(x_{-1}\right)$ (otherwise, either there is $y \triangleright^{*} x$ with $y>x$ or not). Therefore, $x \in X^{0}$ implies $j=1$, hence $y_{1} \in R_{1}\left(x_{-1}\right)=R_{1}\left(y_{-1}\right)$, hence $y \notin X^{0}$, hence $y \succ x$.

(C) Finally, let $x \in X^{1}$; then $y>x$, so $y \succ x$ unless $y \in X^{0}$. If $y_{1} \in R_{1}\left(y_{-1}\right)$, then $y \notin X^{0}$ and we are home; otherwise, $j \neq 1$, hence $y_{1}=x_{1} \in R_{1}\left(x_{-1}\right)$ and $y_{-1}>x_{-1}$. Picking $y_{1}^{\prime} \in R_{1}\left(y_{-1}\right)$, we denote $z_{1}=y_{1}^{\prime} \vee y_{1}$; now $z_{1}>y_{1}$ and $z_{1} \in R_{1}\left(y_{-1}\right)$ by Lemma 3.1. Therefore, $\left(z_{1}, y_{-1}\right) \triangleright^{*}{ }_{1} y$, hence $y \in X^{1}$. 
Lemma 5.2.2. If $x \in X$ is a maximizer for $\bowtie$, then $x$ is a Nash equilibrium (i.e., a maximizer for $\left.\triangleright^{*}\right)$.

Proof. Let $x_{1} \notin R_{1}\left(x_{-1}\right)$; picking $y_{1} \in R_{1}\left(x_{-1}\right)$ appropriately, we obtain $\left(y_{1}, x_{-1}\right) \triangleright_{1} x$, contradicting our assumption. If $x_{1} \in R_{1}\left(x_{-1}\right)$ and there are $i \in N$ and $y_{i}>x_{i}$ such that $\left(y_{i}, x_{-i}\right) \triangleright^{*}{ }_{i} x$, then $x \in X^{1}$ and $\left(y_{i}, x_{-i}\right) \triangleright_{i} x$. If $x_{1} \in R_{1}\left(x_{-1}\right)$ and there is no best response improvement upwards, then $x \in X^{2}$; if $x$ is not a Nash equilibrium, there must be a best response improvement downwards (since all $X_{i}, i \neq 1$, are chains): $y \triangleright^{*} x, y<x$, but then $y \bowtie x$.

The end of the proof is exactly the same as in Theorem 1.

Remark. The proof remains valid without (3.2) and (3.5) provided the statement of Lemma 3.1 holds for every $i \in N$. Unlike Theorem 1 (see Example 1), Theorem 2 only hinges on the monotonicity of the best responses. To the best of our knowledge, however, there is no way to derive the monotonicity without (3.2) and (3.5).

\subsection{Proof of Theorem 3}

Let $\left\{x^{0}, x^{1}, \ldots, x^{m}=x^{0}\right\}$ be a best response improvement cycle. All $x_{2}^{k}(k=0,1, \ldots, m)$ are linearly ordered, hence, by Lemma 3.2, all $x_{1}^{k}$ are linearly ordered too. Without restricting generality, we may assume that $x^{1} \triangleright^{*}{ }_{1} x^{0}$ and $x^{2} \triangleright^{*}{ }_{2} x^{1}$, hence $R_{2}\left(x_{1}^{0}\right) \ni x_{2}^{0}=$ $x_{2}^{1} \notin R_{2}\left(x_{1}^{1}\right), x_{2}^{2} \in R_{2}\left(x_{1}^{1}\right), R_{1}\left(x_{2}^{1}\right) \ni x_{1}^{1}=x_{1}^{2} \notin R_{1}\left(x_{2}^{2}\right)$, and $x_{1}^{3} \in R_{1}\left(x_{2}^{2}\right)$, and that $x_{1}^{1}=x_{1}^{2}$ is maximal among all $x_{1}^{k}$. Applying Lemma 3.1 with $i=2$ and $x_{-i}=x_{1}^{0} \leq x_{1}^{1}=y_{-i}$, we obtain $x_{2}^{0}<x_{2}^{2}$; now applying the same lemma with $i=1$ and $x_{-i}=x_{2}^{0}<x_{2}^{2}=y_{-i}$, we obtain $x_{1}^{1}<x_{1}^{3}$, which contradicts our maximality assumption.

\section{References}

Agliardi E (2000) A generalization of supermodularity, Economics Letters 68: 251-254

Friedman JW, Mezzetti C (2001) Learning in games by random sampling, Journal of Economic Theory 98: 55-84

Fudenberg D, Levine DK (1998) The Theory of Learning in Games. The MIT Press, Cambridge, Mass. - London

Kandori M, Rob R (1995) Evolution of equilibria in the long run: A general theory and applications, Journal of Economic Theory 65: 383-414 
Kukushkin NS (1999) Potential games: A purely ordinal approach, Economics Letters 64: $279-283$

Kukushkin NS (2000) Potentials for binary relations and systems of reactions. Russian Academy of Sciences, Computing Center, Moscow

Kukushkin NS (2004) Best response dynamics in finite games with additive aggregation, Games and Economic Behavior 48: 94-110

Milchtaich I (1996) Congestion games with player-specific payoff functions, Games and Economic Behavior 13: 111-124

Milgrom P, Roberts J (1990) Rationalizability, learning, and equilibrium in games with strategic complementarities, Econometrica 58: 1255-1277

Milgrom P, Roberts J (1991) Adaptive and sophisticated learning in normal form games, Games and Economic Behavior 3: 82-100

Milgrom P, Shannon C (1994) Monotone comparative statics, Econometrica 62: 157180

Monderer D, Shapley LS (1996) Potential games, Games and Economic Behavior 14: $124-143$

Topkis DM (1979) Equilibrium points in nonzero-sum n-person submodular games, SIAM Journal on Control and Optimization 17: 773-787

Vives X (1990) Nash equilibrium with strategic complementarities, Journal of Mathematical Economics 19: 305-321

Vives X (2003) Complementarities and games: New developments, mimeo

Young HP (1993) The evolution of conventions, Econometrica 61: 57-84 